\title{
FATORES QUE INFLUENCIAM A NÃO ADESÃO DA MULHER AO EXAME PAPANICOLAU: REVISÃO DE LITERATURA
}

\author{
FACTORS THAT INFLUENCE WOMEN'S NON-ADHESION TO \\ PAPANICOLAOUEXAMINATION: LITERATURE REVIEW
}

Valdízia Mendes e Silva ${ }^{1}$

Karla Pereira Vasconcelos ${ }^{2}$

Danyelle Dayse de Souza Diniz ${ }^{3}$

Gizele Marinho de Farias ${ }^{4}$

Ana Emília Araújo de Oliveira ${ }^{5}$

RESUMO: OBJETIVO: descrever os fatores que determinam a não adesão das mulheres ao papanicolau. MÉTODO: trata-se de uma revisão de literatura realizada nas bases LILACS, MEDLINE e SCIELO. Foram incluídos na amostra artigos publicados entre os anos de 2010 e 2020 que estavam disponíveis na íntegra. Foram examinados 37 estudos. Após a análise do resumo, a amostra final foi composta por 12 artigos. RESULTADOS: os resultados obtidos foram dispostos e agrupados em quatro categorias pré-estabelecidas: aspectos sociodemográficos das mulheres; sentimentos negativos gerados pela realização do exame; aspectos ginecológicos relacionados a não adesão ao papanicolau; relação da não adesão com o serviço de saúde. Destacaram-se como causa da não adesão: baixa escolaridade, classe social baixa, menores faixas etárias, tabagismo, baixo peso ou obesidade, desconforto, nervosismo, ansiedade, receio, vergonha, constrangimento, medo, tabus e timidez, bem como ideias preconcebidas a respeito do exame e a falta de atitude. Também foramapontados o não uso de métodos contraceptivos, ter 4 ou mais filhos, ausência de sintomas ginecológicos, não possuir relação conjugal, falta de recursos, materiais, espaço e sobrecarga de trabalho, dificuldade de acesso

\footnotetext{
1 Graduanda em Enfermagem pela Universidade Estadual da Paraíba (UEPB), e-mail: valdiziamendes@hotmail.com.

2 Graduanda em Enfermagem pela Universidade Estadual da Paraíba (UEPB), e-mail: karlapv2017@gmail.com.

${ }_{3}$ Graduanda em Enfermagem pela Universidade Estadual da Paraíba (UEPB), e-mail: danyellednz@gmail.com.

Graduanda em Enfermagem pela Uninassau Campina Grande, e-mail: gisellemarinho.10@hotmail.com.

${ }_{5}^{5}$ Mestranda em Ciência e Tecnologia em Saúde pela Universidade Estadual da Paraíba (NUTESUEPB), e-mail: anaemiliaoliveira@hotmail.com.
} 
e insuficiência de vagas, longas filas e marcação aprazada para datas distantes associados às atividades laborais da mulher, falta de oferta profissional, falta de aconselhamento e orientação à paciente, presença do profissional masculino. CONCLUSÃO: observou-se que há diversos fatores que determinam a não adesão das mulheres ao Papanicolau. É de fundamental importância a efetivação de programas que visem a equidade do atendimento, diminuição das iniquidades sociais e oportunização do oferecimento do exame.

Palavras chave: Adesão. Teste de Papanicolau. Saúde da Mulher.

ABSTRACT: OBJECTIVE: to describe the factors that determine women's nonadherence to the pap smear. METHOD: this is a literature review conducted on the LILACS, MEDLINE and SCIELO databases. Articles published between 2010 and 2020 that were available in full were included in the sample. 37 studies were examined. After analyzing the summary, the final sample consisted of 12 articles. RESULTS: the results obtained were arranged and grouped into four pre-established categories: sociodemographic aspects of women; negative feelings generated by the exam; gynecological aspects related to non-adherence to pap smears; relationship of non-adherence with the health service. The following stood out as the cause of nonadherence: low education, low social class, smaller age groups, smoking, underweight or obesity, discomfort, nervousness, anxiety, fear, shame, embarrassment, fear, taboos and shyness, as well as preconceived ideas about regarding the exam and the lack of attitude. It was also pointed out the non-use of contraceptive methods, having 4 or more children, absence of gynecological symptoms, not having a marital relationship, lack of resources, materials, space and work overload, difficulty in access and insufficient spaces, long lines and scheduling scheduled for distant dates associated with the woman's work activities, lack of professional offer, lack of counseling and guidance to the patient, presence of the male professional. CONCLUSION: it was observed that there are several factors that determine women's non-adherence to Pap smears. It is of fundamental importance to put in place programs that aim at equity in care, reduction of social inequities and opportunities to offer the exam.

Keywords: Accession. Papanicolaou test. Women's Health. 


\section{INTRODUÇÃO}

O câncer de colo de útero ou câncer cervical, é causado pela infecção persistente de alguns tipos de Papilomas Humanos (HPV), esses Papilomas são conhecidos como tipos oncogênicos de HPV. As infecções pelo HPV são frequentes e na grande maioria dos casos não causam alterações malignas, no entanto, nos casos que ocorrem alterações celulares, estas podem evoluir para o câncer de colo de útero (INCA, 2020).

O câncer de colo de útero é a terceiro tumor maligno mais frequente em mulheres e a quarta causa de morte da população feminina no Brasil, sendo responsável por 311 mil óbitos por ano no mundo. Segundo informações do Instituto Nacional do Câncer (INCA) estima-se que em 2020 no Brasil haverá 16.590 novos casos de câncer de colo de útero, sendo o exame de papanicolau a principal estratégia para detectar precocemente alterações celulares e até tumores em fases iniciais (INCA, 2020).

O exame preventivo de Papanicolau é uma tecnologia simples, eficaz e de baixo custo para a prevenção do câncer cérvico-uterino e de suas lesões precursoras. Esse exame consiste em um esfregaço de células coletadas da ectocérvice e da endocérvice, que são extraídas do colo do útero por raspagem e em seguida, essas células são dispostas em lâmina de vidro e encaminhada para análise laboratorial. Embora represente $o$ instrumento mais prático e adequado para o rastreamento do câncer de colo de útero a sua cobertura ainda não está dentro dos números preconizados pelo Ministério da Saúde (MS) (OLIVEIRA et al., 2006).

O MS apresenta no Plano de Ações Estratégicas para o Enfrentamento das Doenças Crônicas não Transmissíveis (DCNT) no Brasil, 2011-2022 a estimativa de cobertura de $85 \%$ das mulheres entre 25 a 64 anos, no entanto, dados demonstram que essa taxa ainda não foi atingida. Em 2017 o índice de cobertura chegou a 82,8\%. Segundo o Sistema de Informação do Câncer (SISCAN), em 2019 foram 
realizados no Brasil 6.751.583 exames de colo de útero (BRASIL, 2017; BRASIL, 2019).

Para que seja considerado eficiente, um programa de prevenção de neoplasias cérvico- uterinas deve culminar na diminuição da morbimortalidade, devendo sua cobertura chegar a $85 \%$ na faixa etária preconizada pelo MS. Porém, para que isto aconteça não basta apenas introduzir a oferta dos exames preventivos na Atenção Primária à Saúde (APS), sendo necessário criar meios de mobilizar essas mulheres a comparecerem aos postos de saúde, bem como, utilizar os sistemas de referência para os encaminhamentos pertinentes. Dentre esses meios, a educação em saúde constitui-se de tarefa essencial na atenção básica, a fim de quebrar os estigmas existentes sobre a realização do exame, assim como, orientar sobre a importância da realização do exame de modo precoce para aumentar as chances do prognóstico positivo com o tratamento (SILVA et al., 2018).

Além da educação em saúde, outras intervenções descritas que contribuem para a mobilização de mulheres para coleta do exame incluem: contato telefônico, carta-convite, divulgação na mídia, emprego de agentes de saúde da comunidade, formação de parcerias, rastreio de base populacional e utilização de múltiplas intervenções (SOARES; SILVA, 2016).

Essas intervenções são de suma importância, pois contribuem para 0 acolhimento dessas pacientes, e como consequência para o vínculo com o serviço de saúde, visto que mesmo tendo conhecimento sobre a principal função do exame as mulheres não buscam o serviço para realizá-lo por motivos como vergonha e medo, o que constitui-se de fatores negativos e prejudiciais a continuidade da assistência (SILVA et al., 2018).

Diante do exposto, considerando a alta incidência e mortalidade relacionada aocâncer de colo de útero é de responsabilidade dos gestores e dos profissionais de saúde, emespecial os que estão na APS, considerada porta de entrada das redes de atenção à saúde promover ações que visem a prevenção e controle do câncer de colo de útero utilizando para isso, a detecção precoce por meio da utilização do exame de papanicolau. Deste modo, sabendo da importância do acesso a procedimentos diagnósticos e terapêuticos para prevenção e redução da mortalidade ocasionada por tal neoplasia, o presente artigo tem por objetivo 
descrever os principais fatores que determinam a não adesão de mulheres ao exame de Papanicolau na atenção primária.

\section{METODOLOGIA}

Trata-se de uma revisão de literatura realizada nas bases LILACS, MEDLINE e SCIELO. O processo de obtenção da amostra ocorreu durante o mês de abril de 2020. Para a busca dos artigos foram utilizados os Descritores em Ciências da Saúde (DeCS): Adesão; Papanicolau; Saúde da Mulher. Foram incluídos na amostra artigos publicados no período compreendido entre os anos de 2010 e 2020 que estavam disponíveis na íntegra, escritos em português ou inglês. Excluíram-se da amostra aqueles que se encontravam em duplicidade nas bases, fora do período selecionado, escritos em outros idiomas e os que não atingiam o objetivo deste estudo. Foram examinados 37 estudos, entretanto, após a aplicação dos critérios de exclusão e análise do resumo, a amostra final foi composta por 12 artigos.

\section{RESULTADOS E DISCUSSÕES}

Os artigos obtidos foram dispostos na tabela 1 e posteriormente os resultados obtidos foram dispostos e agrupados em quatro categorias pré-estabelecidas. A primeira categoria se refere aos aspectos sociodemográficos das mulheres, a segunda trata dos sentimentos negativos gerados pela realização do exame, a terceira aborda os aspectos ginecológicos relacionados a não adesão ao papanicolau e a quarta apresenta a relação da não adesão como serviço de saúde. 
Tabela 1- Artigos selecionados para o estudo.

\begin{tabular}{|c|c|c|c|}
\hline TÍTULO DO ARTIGO & AUTORES & OBJETIVO & CONCLUSÃO \\
\hline $\mid \begin{array}{l}\text { Adesão a exames de } \\
\text { saúde recomendados } \\
\text { por mulheres na meia- } \\
\text { idade: dados de um } \\
\text { estudo prospectivo de } \\
\text { mulheres na Austrália }\end{array}$ & $\begin{array}{l}\text { BYLES, J.; LEIGI } \\
\text { L.; CHOJENTA, C } \\
\text { LOXTON, D. }\end{array}$ & \begin{tabular}{|l|} 
Examinar os fatores \\
relacionados aos testes \\
de Papanicolaou, \\
mamografia e teste de \\
colesterol em mulheres \\
australianas de meia- \\
idade com a idade.
\end{tabular} & \begin{tabular}{|l|} 
\\
Saúde e cuidados de saúde \\
são \\
determinantes da triagem.
\end{tabular} \\
\hline $\begin{array}{l}\text { Exame Papanicolau: } \\
\text { fatores que influenciam } \\
\text { a não realização do } \\
\text { exame em mulheres de } \\
40 \text { a } 65 \text { anos }\end{array}$ & $\begin{array}{l}\text { SILVA, J. P.; LEITE, } \\
\text { K. N. S.; SOUZA, T. } \\
\text { A.; SOUSA, K. M. M. } \\
\text { O.; RODRIGUES, S.; } \\
\text { ALVES, J. } \quad \text { P.; } \\
\text { RODRIGUES, A. R. } \\
\text { S.; SOUZA, A. R.D. }\end{array}$ & \begin{tabular}{|l|} 
Caracterizar os fatores \\
que \\
mulheres de 40 a 65 anos \\
de idade a nãorealizarem \\
o examePapanicolau.
\end{tabular} & $\begin{array}{l}\text { O medo do diagnóstico e a } \\
\text { vergonha são os principais } \\
\text { fatores que influenciam a não } \\
\text { adesão das mulheres ao } \\
\text { exame Papanicolau, na faixa } \\
\text { etária estudada. Apesar das } \\
\text { estratégias nacionais para } \\
\text { aumentar a cobertura do } \\
\text { exame, ainda existem essas } \\
\text { dificuldades relacionadas a } \\
\text { realização, influenciando na } \\
\text { baixa cobertura a nível } \\
\text { nacional. }\end{array}$ \\
\hline $\mid \begin{array}{l}\text { Barreiras à realização } \\
\text { do exame Papanicolau: } \\
\text { perspectivas } \\
\text { usuárias e profissionais } \\
\text { da Estratégia de Saúde } \\
\text { da Família da cidade de } \\
\text { Vitória da Conquista-BA }\end{array}$ & $\begin{array}{l}\text { AGUILAR, R. } \\
\text { SOARES, D.A. }\end{array}$ & 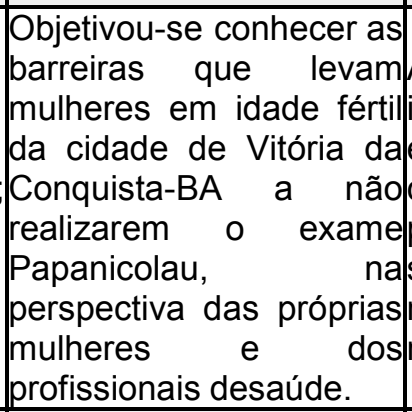 & $\begin{array}{l}\text { A congruência } \\
\text { incongruência de significados } \\
\text { emergidos possibilitarão um } \\
\text { caminho que transforme a } \\
\text { prática do profissional de } \\
\text { saúde e atendimento das } \\
\text { necessidades dessas } \\
\text { mulheres. }\end{array}$ \\
\hline \begin{tabular}{|l} 
Detecção precoce \\
prevenção do câncer de \\
colo uterino: saberes e \\
práticas educativas
\end{tabular} & 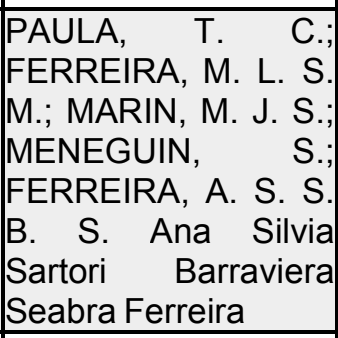 & \begin{tabular}{|l|} 
Apreender os saberes de \\
mulheres sobre a \\
prevenção do câncer de \\
colo uterino por meio do \\
exame de Papanicolaou.
\end{tabular} & \begin{tabular}{|l} 
Depreende-se que a falta de \\
conhecimento pode intervir \\
na adesão ao exame de \\
Papanicolaou pous \\
mulheres, destacando- se a \\
importância da prática \\
educativa como ferramenta \\
nessa prevenção. \\
\end{tabular} \\
\hline \begin{tabular}{|l} 
Fatores associados a \\
não adesão ao ao \\
Papanicolau entre \\
mulheres atendidas pela \\
Estratégia
\end{tabular} & $\begin{array}{l}\text { ANDRADE, M. } \\
\text { ALMEIDA, M. M. G.; } \\
\text { ARAÚJO, T. } \\
\text { SANTOS, K. O. }\end{array}$ & $\begin{array}{l}\text { Analisar fatores } \\
\text { associados a não adesão } \\
\text { ao Papanicolau em } \\
\text { mulheres de 25 a } 59\end{array}$ & $\begin{array}{l}\text { Houve elevada cobertura de } \\
\text { realização do Papanicolau; } \\
\text { entretanto, mulheres }\end{array}$ \\
\hline $\begin{array}{l}\text { Saúde da Família em } \\
\text { Feira de Santana,Bahia, } \\
2010\end{array}$ & & $\begin{array}{l}\text { anos de idade atendidas } \\
\text { pela Estratégia Saúde da } \\
\text { Familia em Feira de } \\
\text { Santana, Bahia, Brasil, } \\
\text { em 2010. }\end{array}$ & $\begin{array}{l}\text { com fatores de risco para o } \\
\text { desenvolvimento daneoplasia } \\
\text { cérvico- uterina, como pouca } \\
\text { escolaridade e multiparidade, } \\
\text { apresentaram cobertura } \\
\text { abaixo do esperado. }\end{array}$ \\
\hline
\end{tabular}




\begin{tabular}{|c|c|c|}
\hline 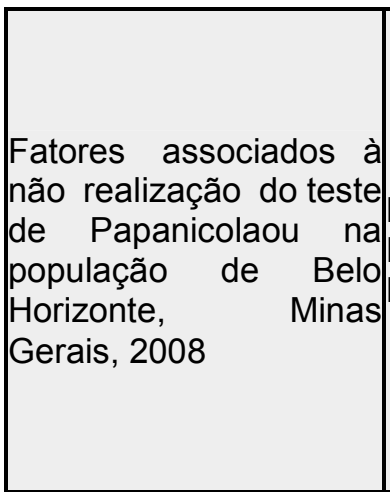 & 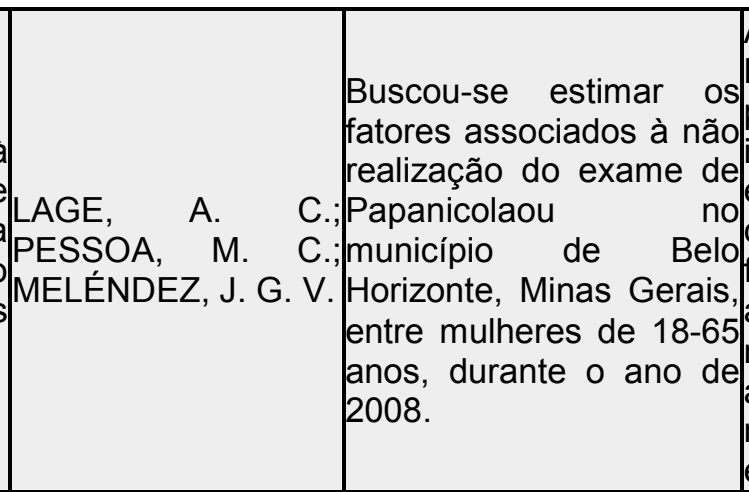 & $\begin{array}{l}\text { A prevalência do exame em } \\
\text { Belo Horizonte foi satisfatória, } \\
\text { porém insuficiente para } \\
\text { impactar no perfil } \\
\text { epidemiológico do câncer do } \\
\text { colo uterino. E preciso } \\
\text { fortalecer e qualificar as } \\
\text { ações de promoção da saúde } \\
\text { necessárias para mais } \\
\text { adesão das mulheres que } \\
\text { não estão realizando o } \\
\text { exame dePapanicolaou. }\end{array}$ \\
\hline $\begin{array}{lr}\text { Não adesão r às } \\
\text { diretrizes } & \text { para } \\
\text { rastreamento do câncer } \\
\text { do colo do útero entre } \\
\text { mulheres re rue } \\
\text { frequentaramo pré-natal }\end{array}$ & 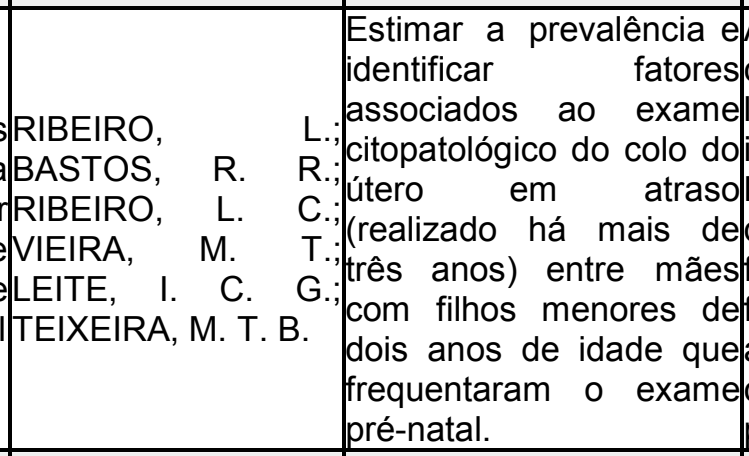 & $\begin{array}{l}\text { A prevalência de exame } \\
\text { citopatológico atualizado está } \\
\text { ligeiramente abaixo do } \\
\text { indicado pela Organização } \\
\text { Mundial da Saúde. Além } \\
\text { disso, o fato de ter } \\
\text { frequentado o pré-natal não } \\
\text { foi determinante para garantir } \\
\text { a realização do exame } \\
\text { citopatológico segundo } \\
\text { periodicidade recomendada. }\end{array}$ \\
\hline $\begin{array}{l}\text { Percepção de usuárias } \\
\text { acerca do exame de } \\
\text { detecção precoce do } \\
\text { câncer de colo uterino }\end{array}$ & \begin{tabular}{|l|l|} 
NEVES, K. T. Q.; & Objetivou-se descrever a \\
OLIVEIRA, A. W. W.; \\
GALVÃO, T. R. A. F.; \\
Fercepção de usuárias \\
FERREIRA, I. T.; \\
MANGerca do exame de \\
MANGANE, E. M.; \\
SOUSA, L. B.
\end{tabular} & $\begin{array}{l}\text { Conclui-se que as } \\
\text { entrevistadas percebem o } \\
\text { exame como importante e } \\
\text { que eventuais motivos para a } \\
\text { recusa podem ser evitados } \\
\text { por meio do fortalecimento de } \\
\text { vínculos no serviço. }\end{array}$ \\
\hline $\begin{array}{l}\text { Percepção e adesão } \\
\text { das mulheres quanto ao } \\
\text { exame citopatológico }\end{array}$ & 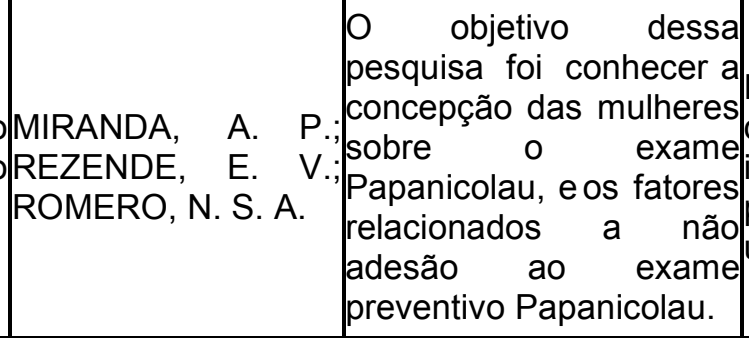 & $\begin{array}{l}\text { Foi observado que a maioria } \\
\text { das mulheres entenderam a } \\
\text { importância da promoção e } \\
\text { prevenção do câncer colo de } \\
\text { útero. }\end{array}$ \\
\hline 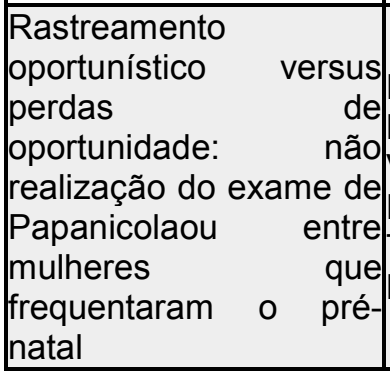 & 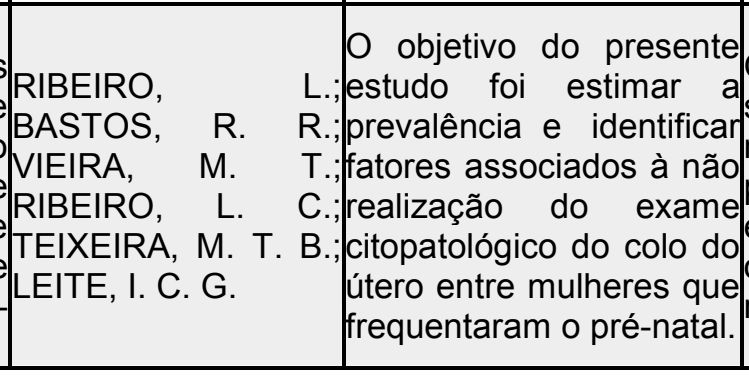 & $\begin{array}{l}\text { contato com o serviço } \\
\text { saúde para realização do pr } \\
\text { natal não foi determinan } \\
\text { para garantir o acesso } \\
\text { exame, indicando perda } \\
\text { oportunidades onde } \\
\text { rastreamento é oportunístic }\end{array}$ \\
\hline
\end{tabular}




\begin{tabular}{|c|c|c|c|}
\hline $\begin{array}{l}\text { Tendo que se submeter } \\
\text { ao exame Papanicolau } \\
\text { regularmente: uma } \\
\text { análise sob a ótica da } \\
\text { desmedicalização }\end{array}$ & OLIVEIRA, D.S. & $\begin{array}{l}\text { Os objetivos deste } \\
\text { Os estudo são: identificar os } \\
\text { significados do exame } \\
\text { Papanicolau anal para } \\
\text { mulheres e analisar o } \\
\text { processo de interação } \\
\text { social dessas mulheres } \\
\text { com o exame, no } \\
\text { contexto do câncer do } \\
\text { colo doútero, a partir dos } \\
\text { significados por elas } \\
\text { atribuídos. } \\
\end{array}$ & $\begin{array}{l}\text { Conclui-se que, mesmo que } \\
\text { não seja de forma regular } \\
\text { como preconizado pelos } \\
\text { protocolos, a mulher se } \\
\text { submete ao exame } \\
\text { Papanicolau para prevenção } \\
\text { de doenças. Ficou evidente, } \\
\text { pelos dados, que é } \\
\text { necessário promover } \\
\text { atividades que vão além de } \\
\text { orientações e informações } \\
\text { sobre o câncer do colo do } \\
\text { útero e a importância deste } \\
\text { exame. É importante criar } \\
\text { estratégias de promoção da } \\
\text { saúde para estimular a } \\
\text { autonomia das mulheres e o } \\
\text { empoderamento do próprio } \\
\text { corpo, para, assim, propiciar } \\
\text { maior cobertura do exame } \\
\text { através da adesão regular } \\
\text { das mulheres. }\end{array}$ \\
\hline $\begin{array}{l}\text { Conhecimento das } \\
\text { mulheres sobre o risco } \\
\text { de câncer cervical } \\
\text { fatores, triagem } \\
\text { motivos da não } \\
\text { participação na triagem } \\
\text { de câncer cervical } \\
\text { programa na Estônia }\end{array}$ & $\begin{array}{l}\text { KIVISTIK, A.; LANG, } \\
\text { K.; BAILI, P.; } \\
\text { ANTTILA, } \\
\text { VEERUS, P. }\end{array}$ & \begin{tabular}{|l} 
Identificar \\
conscientização dos \\
fatores de risco de \\
câncer do colo do útero, \\
razões pelas quais as \\
mulheres não querem \\
participar do programa \\
de triagem cervical e \\
deseja uma melhor \\
organização ran do \\
programa.
\end{tabular} & $\begin{array}{l}\text { As mulheres precisam de } \\
\text { mais informações sobre os } \\
\text { fatores de risco de câncer do } \\
\text { colodo útero e o programa de } \\
\text { triagem. Elas preferem } \\
\text { compartilhamento de } \\
\text { informações endereçadas } \\
\text { pessoalmente. Grupos } \\
\text { minoritários devem ser } \\
\text { abordados em seu próprio } \\
\text { idioma. Também } \\
\text { necessária uma melhor } \\
\text { colaboração com os } \\
\text { provedores de serviços e } \\
\text { manchas desanimadoras fora } \\
\text { doprograma. }\end{array}$ \\
\hline
\end{tabular}

\section{I - Aspectos sociodemográficos}

O exame citopatológico é uma ferramenta efetiva na prevenção ao câncer do colo do útero. Apesar da facilidade ao acesso, muitas mulheres deixam de realizar o exame. Um dos fatores apontados na literatura para este feito é a baixa escolaridade das mulheres. Estudos apresentam associação significativa entre o tempo de estudo e a adesão ao papanicolau, de modo que quanto menor o índice de escolaridade, menor a adesão ao exame e mais acentuada é a dificuldade na 
compreensão das medidas preventivas e de controle da saúde (SILVA et al., 2018; AGUILAR; SOARES, 2015; LAGE; PESSOA; MELÉNDEZ; 2012; ANDRADE et al., 2014; RIBEIRO et al., 2016).

Andrade et al. (2014) demonstram que mulheres com menor escolaridade têm maior risco para o desenvolvimento de neoplasia cérvico-uterina. Além disso, este público desconhecia o objetivo do exame. Uma vez que não se entende a importância da realizaçãodo exame, tende-se a não compreender a associação do mesmo a uma prática de saúde, bem como a necessidade de realização periódica (AGUILAR; SOARES, 2015; SILVA et al., 2018).

O estudo de Silva et al. (2018) demonstrou que as mulheres de classe social mais baixa procuram menos os serviços de saúde para prevenção de doenças. Segundo os autores, elas têm maior busca por procedimentos curativos por não compreenderem a importância dos métodos preventivos.

Em relação à idade, alguns estudos apontam que mulheres com menores faixas etárias, entre 18 e 24 anos, foram as que menos realizaram o Papanicolau. Segundo uma análise bivariada esta faixa etária têm menores chances de realizar o exame comparada ao grupo com idade entre 25 a 35 anos. Embora a faixa etária preconizada pelo Ministério da Saúde compreenda o público entre 25 e 35 anos, é ideal que se fortaleça a necessidade da adesão ao exame por parte das adolescentes, pois apesar de que as atipias citológicas nesta população sejam regularmente de baixo grau, há risco de progressão para as lesões não tratadas, podendo chegar a um carcinoma, principalmente quando se trata de infecções por HPV oncogênico (LAGE; PESSOA; MELÉNDEZ; 2012; RIBEIRO et al., 2016).

Segundo um estudo australiano, mulheres tabagistas, com baixo peso ou obesidade eram mais propensas a não realizarem o papanicolau (BYLES et al., 2014). Já no Brasil, não foi encontrada associação entre o tabagismo e a não adesão ao exame (LAGE; PESSOA; MELÉNDEZ; 2012). 


\section{II - Sentimentos negativos gerados pela realização do exame}

O desconforto, nervosismo, ansiedade e receio são frequentemente apontados nos estudos. A vergonha é um dos sentimentos mais comumente relatados na literatura como motivo para a não adesão ao papanicolau. Por ser uma prática de saúde que envolve um procedimento invasivo, que remete à sexualidade e exposição do corpo, pode provocar sentimentos negativos como vergonha e constrangimento diante do profissional. A perda de autonomia e fragilidade diante do procedimento, atrelado a fatores como tabus e timidez podem exacerbar tais sentimentos, provocando desconfortos e dor durante o exame (SILVA et al., 2018; AGUILAR; SOARES, 2015; PAULA et al; 2019; MIRANDA; REZENDE; ROMERO, 2018; OLIVEIRA, 2018).

É importante frisar que grande parte das mulheres que não realizam o exame ou não têm total adesão ao mesmo possuem ideias preconcebidas sobre o papanicolau a partir de experiências negativas vivenciadas por outras usuárias. Além disso, um fator de grande impacto é a falta de atitude para a realização do exame citológico, decorrente da crença de que o câncer de colo do útero é uma doença distante da sua realidade. Por diversas vezes, este pensamento é modificado apenas no momento em que elas ou pessoas próximas desenvolvem a doença (AGUILAR; SOARES, 2015).

Outro sentimento de grande destaque é o medo do resultado, quer seja por uma possível doença sexualmente transmissível (DST) ou câncer, doença que carrega consigo oestigma pela sua capacidade de em casos extremos, comprometer a vida (SILVA et al., 2018; AGUILAR; SOARES, 2015; PAULA et al; 2019; OLIVEIRA, 2018; KIVISTIK et al., 2011). 


\section{III - Aspectos ginecológicos relacionados à não adesão ao papanicolau}

Em relação aos aspectos ginecológicos, foi encontrada relação entre o não uso de métodos contraceptivos e a não adesão ao Papanicolau. Além disso, houve associação entre ter quatro ou mais filhos e a não realização periódica do exame, o que sugere a ligação com o encargo dos cuidados familiares, contribuindo para que a mulher deixe o autocuidado para segundo plano (ANDRADE et al., 2014).

A ausência de sintomas ginecológicos também foi apontada como uma causa, demonstrando a concepção de que o serviço de saúde só deve ser procurado após o estabelecimento de alguma doença ou aparecimento de sintomas, comportamento este presente nos países em desenvolvimento (ANDRADE et al., 2014; OLIVEIRA, 2018; RIBEIRO et al., 2016).

Quanto à relação conjugal, constatou-se que as mulheres solteiras têm menoreschances de realizar o citológico quando comparadas às casadas. Este dado aponta uma associação entre atividade sexual e realização do papanicolau, de modo que o exame preventivo ocorre em momentos oportunos do planejamento familiar e assistência ginecológica e obstétrica. Desta forma, mulheres que vivem com companheiro estão mais propensas à práticas preventivas (LAGE; PESSOA; MELÉNDEZ; 2012; RIBEIRO et al., 2016).

\section{IV - Relação da não adesão com o serviço de saúde}

Além das dificuldades relativas à pessoa, a literatura apontou limitações relacionadasaos serviços de saúde. Trata-se da vulnerabilidade programática, que envolve os camposdo acesso aos serviços de saúde, forma de organização destes, o vínculo usuário- profissional, tal como suas ações e recursos na área de abrangência do serviço de saúde (BERTOLOZZI et al., 2009). 
A falta de recursos, materiais e espaço, assim como a sobrecarga de trabalho nos serviços de saúde são apontados como barreiras que afetam a oferta de vagas para a realização do exame. A dificuldade de acesso, bem como a insuficiência de vagas para marcação de consulta mostram que as necessidades de saúde sexual deste público não estão sendo supridas (AGUILAR; SOARES, 2015).

Longas filas e marcação aprazada para datas distantes são fatores limitantes daadesão à medida que impactam diretamente nas atividades laborais da mulher. A soma do trabalho e do cuidado familiar sobrecarrega e dificulta sua participação ativa das práticas preventivas, tendo em vista a incompatibilidade de horários entre os horários ofertados pelos serviços de saúde e a jornada de trabalho, resultando na não realização do papanicolau (AGUILAR; SOARES, 2015; KIVISTIK et al., 2011; OLIVEIRA, 2018).

Embora preconizado pelo Ministério da Saúde, existem mulheres que não realizaram o citológico do colo do útero no período de pré-natal, dentre elas, algumas nunca haviamse submetido ao exame. Considerando uma média de 6,94\% de consultas de pré-natal realizadas pelas participantes do estudo, observa-se perda de oportunidades por falta de oferta da parte profissional (RIBEIRO et al., 2013; RIBEIRO et al., 2016).

Outro agente é a falta de orientação de qualidade do profissional à paciente. Parte da população feminina desconhece a principal função do exame, contribuindo para a não adesão na realização a este. O profissional deve educar e aconselhar para ampliar o conhecimento da mulher, possibilitando que compreenda a importância da prevenção e adesão ao exame (PAULA et al; 2019; SILVA et al., 2018).

Com relação aos recursos humanos para realização do papanicolau, a presença do profissional masculino mostrou impacto negativo na adesão e agravamento dos sentimentos negativos relativos ao exame. As relações de gênero socialmente construídas definem ações de moralidade relacionadas ao corpo e sexualidade, de modo que a exposição e manipulação da genitália feminina por parte do profissional do sexo masculinopode ser considerado um ato de imoralidade, tornando-se um motivo de não realizarem o exame (AGUILAR; SOARES, 2015; NEVES et al., 2016; MIRANDA; REZENDE; ROMERO, 2018; OLIVEIRA, 2018). 
Neste sentido, o vínculo profissional-paciente demonstra-se fundamental para o bem-estar das mulheres durante a consulta ginecológica. O profissional de enfermagem deve atuar desvendando os medos e receios da usuária através do diálogo, assim como promovendo estratégias de educação em saúde para apropriar a mulher do conhecimento acerca do exame. Estas ações contribuem para a adesão ao exame e colaboram para apercepção positiva do procedimento (NEVES et al., 2016).

\section{CONCLUSÃO}

Ao fim da pesquisa, observou-se que há diversos fatores que determinam a não adesão das mulheres ao Papanicolau, desde aspectos sociodemográficos até barreiras relacionadas ao serviço de saúde. Compreender as limitações associadas à não realização periódica do exame constitui o primeiro passo para o estabelecimento de medidas oportunas para cada realidade.

Dentre os aspectos sociodemográficos destacaram-se como causa da não adesão a baixa escolaridade, classe social baixa, menores faixas etárias, ser tabagista, ter baixo peso ou obesidade. Tais elementos permitem refletir que se fazem necessárias ações para minimização das iniquidades sociais e consequente aumento da cobertura do exame.

Sentimentos negativos como desconforto, nervosismo, ansiedade, receio, vergonha, constrangimento, medo, tabus e timidez também estiveram associados, bem como ideias preconcebidas a respeito do exame e a falta de atitude para realizá-lo. Deste modo, é imprescindível a garantia de informações de qualidade adequadas à realidade das usuárias através da educação em saúde, a fim de promover empoderamento e autonomia dasmulheres, oportunizando a realização do papanicolau.

Quanto aos aspectos ginecológicos foram apontados o não uso de métodos contraceptivos, ter 4 ou mais filhos, ausência de sintomas ginecológicos e não possuir relação conjugal. Pode-se inferir que o comportamento destas mulheres 
demonstra a distância da sua realidade com a realização de práticas preventivas. Em vista disso, sugere- se o desenvolvimento de ações de cunho educativo que estimulem a modificação do estilo de vida por meio da aproximação entre as mulheres e métodos preventivos de saúde.

Em relação aos fatores associados ao serviço de saúde destacaram-se a falta de recursos, materiais, espaço e sobrecarga de trabalho, dificuldade de acesso e insuficiência de vagas, longas filas e marcação aprazada para datas distantes associados às atividades laborais da mulher, falta de oferta profissional, falta de aconselhamento e orientação à paciente, presença do profissional masculino. Diante disso, salienta-se a relevância da construção de vínculo entre serviço e usuárias, assim como implantação de intervenções que objetivem a inserção das usuárias para atendimento humanizado e equitativo.

Portanto conclui-se que não são necessárias apenas medidas educativas, mas compreender mais profundamente as necessidades de cada realidade para que as barreiras associadas a não realização do papanicolau sejam minimizadas. É de fundamental importância a efetivação de programas que visem a equidade do atendimento, diminuição das iniquidades sociais e oportunização do oferecimento do exame.

\section{REFERÊNCIAS BIBLIOGRÁFICAS}

AGUILAR, R. P.; SOARES, D. A. Barreiras à realização do exame Papanicolau: perspectivas de usuárias e profissionais da Estratégia de Saúde da Família da cidade de Vitória da ConquistaBA. Physis Revista de Saúde Coletiva, Rio de Janeiro, v. 25, n. 2, p.359-379, 2015.

ANDRADE, M. S. et al. Fatores associados à não adesão ao Papanicolau entre mulheres atendidas pela Estratégia Saúde da Família em Feira de Santana, Bahia, 2010. Epidemiologia e Serviços de Saúde, Brasília, v. 23, n. 1, p. 111-120, 2014.

BERTOLOZZI, M. R. et al. Os conceitos de vulnerabilidade e adesão na Saúde Coletiva. Revista Escola de Enfermagem da USP, v. 43, n. 2, p. 1326-1330, 2009.

BRASIL. Ministério da Saúde. Plano de Ações Estratégicas para o Enfrentamento das Doenças Crônicas Não Transmissíveis (DCNT). Brasília: Ministério da Saúde, 2017. Disponível em: https://www.saude.gov.br/vigilancia-em-saude/vigilancia-de-doencas- cronicasnao-transmissiveis-dcnt/plano-de-acoes-estrategicas-para-o-enfrentamento-das- doencascronicas-nao-transmissiveis-dcnt. Acesso em: 30 de abril de 2020.

BRASIL. Ministério da saúde. Secretaria de Vigilância em Saúde. Departamento de Informática 
do SUS (Datasus). Sistema de Informação do Câncer de Colo de Útero - SISCOLO/SISCAN. Brasília: Ministério da Saúde, 2019. Disponível em: http://tabnet.datasus.gov.br/cgi/dhdat.exe?SISCAN/cito_colo_pacbr.def . Acesso em: 25de abril de 2020.

INCA. Instituto Nacional do Câncer. Câncer de colo de útero. 2020. Disponível em: https://www.inca.gov.br/tipos-de-cancer/cancer-do-colo-do-utero. Acesso em 25 de abrilde 2020.

BYLES, J. et al. Adherence to recommended health checks by women in mid-life: data from a prospective study of women across Australia. Australian and New Zealand Journal of Public Health, v. 38, n. 1, p. 39-43, 2014.

KIVISTIK, A. et al. Women's knowledge about cervical cancer risk factors, screening, and reasons for nonparticipation in cervical cancer screening programme in Estonia. BioMed Central Women's Health, v. 11, n. 43, 2011.

LAGE, A. C.; PESSOA, M. C.; MELÉNDEZ, J. G. V. Fatores associados à não realização do teste de papanicolau na população de Belo Horizonte, Minas Gerais, 2008. Revista Mineira de Enfermagem, v. 17, n. 3, p. 565-570, 2013.

MIRANDA, A. P.; REZENDE, E. V.; ROMERO, N. S. A. Percepção e adesão das mulheres quanto ao exame citopatológico. Revista Nursing, v. 21, n. 246, p. 2435-2438,2018.

NEVES, K. T. Q. et al. Percepção de usuárias acerca do exame de detecção precoce docâncer de colo uterino. Cogitare Enfermagem, v. 21, n. 4, p. 01-07, 2016.

OLIVEIRA, D. S. Tendo que se submeter ao exame Papanicolau regularmente: uma análise sob a ótica da desmedicalização. Dissertação (Mestrado em Enfermagem, Saúde e Sociedade) - Faculdade de Enfermagem da Universidade do estado do Rio de Janeiro. Riode Janeiro, p. 61, 2018.

OLIVEIRA, M. M. H. N. et al. Cobertura e fatores associados à não realização do exame preventivo de Papanicolaou em São Luís, Maranhão. Revista Brasileira de Epidemiologia. v. 9, n. 2, p. 325-34, 2006.

PAULA, T. C. et al. Detecção precoce e prevenção do câncer de colo uterino: saberes epráticas educativas. Enfermagem em Foco, v. 10, n. 2, p. 47-51, 2019.

RIBEIRO, L. et al. Não adesão às diretrizes para rastreamento do câncer do colo do úteroentre mulheres que frequentaram o pré-natal. Revista Brasileira de Ginecologia e Obstetrícia, v. 35, n. 7, p. 323-330, 2013.

RIBEIRO, L. et al. Rastreamento oportunístico versus perdas de oportunidade: não realização do exame de Papanicolaou entre mulheres que frequentaram o pré-natal. Caderno de Saúde Pública, Rio de Janeiro, v. 32, n. 6, e00001415, 2016.

SILVA, J. P. et al. Exame Papanicolau: fatores que influenciam a não realização do exameem mulheres de 40 a 65 anos. Arquivos de Ciências da Saúde, v. 25, n. 2, p. 15-19, 2018.

SOARES, M. B. O.; SILVA, S. R. Intervenções que favorecem a adesão ao exame de colpocitologia oncótica: revisão integrativa. Revista Brasileira de Enfermagem. v. 69, n.2, p. 404-14, 2016. 